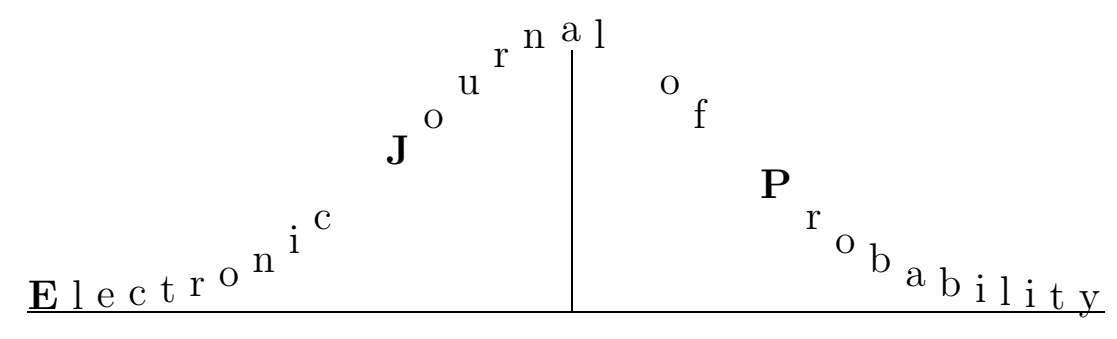

Correction to Vol. 3 (1998), Paper no. 3, pages 1-34

Journal URL http://www.math.washington.edu/〜 ejpecp/

\title{
Errata to \\ Fluctuations of a Surface Submitted to a Random Average Process
}

There follow corrections to Section 3 of Ferrari and Fontes (1998). We thank Beat Niederhauser for pointing out to us a mistake in (3.14) of that publication.

The first correction concerns an assertion in the first full paragraph of page 13 . The one but last sentence starting "The second moment condition..." should end "... is then seen to be recurrent for $d=1$ and 2 , and transient for $d \geq 3$ ".

The remaining corrections are for arguments in the proof of Lemma 3.2. One (in (3.11)) is just an inversion. The others originate in a misquote to Spitzer (1964). They all concentrate in the paragraphs of equations (3.11)-(3.18). The correct paragraphs should read as follows.

"Thus,

$$
\frac{f(s)}{g(s)}=\left[\frac{1+(1-\gamma) s \phi_{T}(s)}{1+\left(1-\gamma^{\prime}\right) s \phi_{\tilde{T}}(s)}\right]^{-1}=\left[\frac{\left[1 / \phi_{\tilde{T}}(s)\right]+(1-\gamma) s\left[\phi_{T}(s) / \phi_{\tilde{T}}(s)\right]}{\left[1 / \phi_{\tilde{T}}(s)\right]+\left(1-\gamma^{\prime}\right) s}\right]^{-1} .
$$

Now,

$$
\phi_{T}(s)=\sum_{x \neq 0} \phi_{T_{x}}(s) p_{x}, \quad \phi_{\tilde{T}}(s)=\sum_{x \neq 0} \phi_{T_{x}^{\prime}}(s) p_{x}^{\prime} \stackrel{*}{=} \sum_{x \neq 0} \phi_{T_{x}}(s) p_{x}^{\prime}
$$

where $T_{x}$ is the hitting time of the origin of the process $D_{n}$ starting at $x$, and $p_{x}, x \neq 0$, is the distribution of the jump from the origin of the same process. $T_{x}^{\prime}$ and $p_{x}^{\prime}$ are the analogues of $T_{x}$ and $p_{x}$ for the process $H_{n}$. Also, $\phi_{T_{x}}=\sum_{n \geq 0} \mathbf{P}\left(T_{x}>n\right) s^{n}$, and similarly for $\phi_{T_{x}^{\prime}}$. Since $T_{x}$ and $T_{x}^{\prime}$ have the same distribution for all $x \neq 0$, we have that $\phi_{T_{x}}=\phi_{T_{x}^{\prime}}$ for all $x \neq 0$, and the identity $*$ above is justified.

Now, we have, on the one hand, that

$$
\lim _{s \rightarrow 1} \phi_{\tilde{T}}(s)=\mathbf{E}(\tilde{T})=\infty
$$


and, on the other hand, by P32.1 on p.378 and P32.2 on p.379 of Spitzer (1964), that

$$
\lim _{s \rightarrow 1} \phi_{T_{x}}(s) / \phi_{\tilde{T}_{0}}(s)=a(x),
$$

for all $x$, where $\tilde{T}_{0}=\inf \left\{n \geq 1: H_{n}=0\right\}, \phi_{\tilde{T}_{0}}(s)=\sum_{n \geq 0} \mathbf{P}\left(\tilde{T}_{0}>n \mid H_{0}=0\right) s^{n}$ and $a$ is defined in Chapter VII of Spitzer (1964).

We have that $a$ is integrable with respect to $\left(p_{x}\right)$ and $\left(p_{x}^{\prime}\right)$ in all dimensions. For $d \geq 3$, this follows from the boundedness of $a$ (which we let the reader check). In $d=1$ and 2, it follows from the bounds on $a(x)$, in terms of $|x|$, in P12.3 and P28.4 of Spitzer (1964), pp.124 and 345 , respectively, and the fact that $\left(p_{x}\right)$ and $\left(p_{x}^{\prime}\right)$ both have absolute first moments (this follows from assumption (2.1)).

To be able to apply the dominated convergence theorem to conclude that

$$
\lim _{s \rightarrow 1} \frac{f(s)}{g(s)}=\frac{\left(1-\gamma^{\prime}\right) \sum_{x} a(x) p_{x}^{\prime}}{(1-\gamma) \sum_{x} a(x) p_{x}}
$$

we need to find $b(\cdot)$ integrable with respect to $\left(p_{x}\right)$ and $\left(p_{x}^{\prime}\right)$ such that

$$
\phi_{T_{x}}(s) / \phi_{\tilde{T}_{0}}(s) \leq b(x)
$$

for all $x \neq 0$ and $s<1$. For that, let $\mathcal{N}$ denote the set of nearest neighboring sites to the origin. Let

$$
\begin{aligned}
\wp & :=\min _{e \in \mathcal{N}} \gamma_{H}(0, e), \\
\tau & :=\max _{e \in \mathcal{N}} T_{e} .
\end{aligned}
$$

By $(2.2), \wp>0$. Notice first that

$$
\phi_{T_{x}}(s) / \phi_{\tilde{T}_{0}}(s) \leq\left(\phi_{T_{x}}(s) / \wp\right) \sum_{e \in \mathcal{N}} \phi_{T_{e}}(s) .
$$

$"$

To conclude, we remark that, since $p_{x}=\gamma(0, x) /(1-\gamma)$ and $p_{x}^{\prime}=\gamma_{H}(0, x) /\left(1-\gamma^{\prime}\right), x \neq 0$, the right hand side of (3.14) becomes $\sum_{x} \gamma_{H}(0, x) a(x) / \sum_{x} \gamma(0, x) a(x)$. By the definition of $a$ and the Markov property of $H_{n}$, it can be shown that $\sum_{x} \gamma_{H}(0, x) a(x)=1$. Thus, finally,

$$
\lim _{s \rightarrow 1} \frac{f(s)}{g(s)}=\left[\sum_{x} \gamma(0, x) a(x)\right]^{-1} .
$$

\section{References}

- P.A. Ferrari, L.R.G. Fontes (1998), Fluctuations of a surface submitted to a random average process, Electronic Journal of Probability 3, paper \#6. 
- F. Spitzer (1964) Principles of Random Walk, Academic Press.

Instituto de Matemática e Estatística - Universidade de São Paulo Rua do Matão 1010 - Cidade Universitária 05508-090 São Paulo - SP — Brasil <pablo@ime.usp.br>, <lrenato@ime.usp.br> 